\title{
OTTO BAUER ET LA QUESTION DES NATIONALITÉS
}

\section{OTTO BAUER AND THE QUESTION OF NATIONALITIES}

\author{
FLORIAN GULLI \\ Université de Franche-Comté
}

\section{RÉSUMÉ}

Le texte se propose d'expliciter la définition de la nation proposée par Otto Baver dans La question des nationalités (1907). Il s'agit de partir du concept de caractère national pour remonter à celui de « communauté de destin ». Ce dernier, pensé dans le cadre de la conception matérialiste de l'historie développée par Marx, désigne l'interaction constante, la communication directe et indirecte des co-nationaux. La nation n'est pas d'abord l'État ou un territoire économique donné, mais une interaction permanente médiatisée par des « bien culturels ». Cette proposition constitue un enrichissement de la conception marxiste de l'histoire ; elle y intègre les facteurs culturels et psychiques souvent éludés. Elle les prend en charge sans jamais les laisser s'autonomiser en lecture culturaliste ou psychologique de l'histoire.

Mots-clefs : nation, classe, marxisme, communauté, identité

\begin{abstract}
This text aims at explaining the way Otto Baver defines nation in The question of nationalities. He starts from the concept of national character and traces back to the concept of common destiny. The latter conceived within Marx's materialistic theory of history, expresses the constant interaction, the direct and indirect communication of co-nationals. Nation is not only the state or a given economic territory, but a permanent interaction mediated by « cultural possessions ». This proposition constitutes an enrichment of the marxist theory of history in which are integrated cultural and psychic factors often not taken into account. They are never treated as an abstraction, as is the case of a cultural or psychological reading of history.
\end{abstract}

Keywords: Nation, Class, Marxism, Community, Identity 


\section{RESUM \\ Otto Bauer i la qüestió de les nacionalitats}

Aquest text es proposa explicitar la definició de la nació proposada per Otto Baver a La qüestió de les nacionalitats (1907). Es tracta de partir del concepte de caràcter nacional per a arribar al de "comunitat de desti". Aquest darrer, plantejat dins el marc de la concepció materialista de la història desenvolupada per Marx, designa la interacció constant, la comunicació directa i indirecta dels co-nacionals. La nació no és per principi l'Estat o un territori econòmic determinat, sinó una interacció permanent mediatitzada pels "béns culturals". Aquesta proposta constitueix un enriquiment de la concepció marxista de la història; integra els factors culturals i psicològics molt a sovint evadits. Els presta atenció sense deixar-los fer-se autònoms des d'una lectura culturalista o psicològica de la història.

Paraules clau: nació, classe, marxisme, comunitat, identitat 


\section{Contexte}

Otto Baver (1881-1938) est un théoricien quelque peu oublié aujourd'hui. Associé à ce que l'on nomme parfois I' "austromarxisme », mêlant à chaque instant philosophie et sciences empiriques, il fut en même temps dirigeant du parti social-démocrate autrichien. Baver appartient à cette catégorie d'intellectuels marxistes d'avant la grande guerre qui ont besoin de connaissances empiriques pour résoudre des problèmes concrets et prendre des décisions. Ces connaissances nourrissent en retour leurs réflexions théoriques. Ce type de position intellectuelle est d'importance. Il détourne la théorie de l'indétermination abstraite. Trop souvent, y compris dans ce que deviendra le marxisme après la première guerre mondiale, ${ }^{1}$ la théorie est déconnectée des enjeux politiques de son temps. Et quand il lui arrive de $s^{\prime} y$ rapporter, il est toujours très difficile d'en dégager les implications politiques concrètes. Bien souvent, on ne saurait déduire aucune décision des philosophèmes proposés par les théoriciens, ou alors les décisions les plus contradictoires entre elles.

Si Otto Baver s'intéresse à la question des nationalités, c'est donc pour résoudre une difficulté théorique et pratique et pour aider à la constitution d'un programme politique qui orientera l'activité du parti social-démocrate autrichien. Pourquoi écrire, en 1907, La question des nationalités ? II faut rappeler que l'Autriche est alors un empire, l'empire austro-hongrois.

1 Voir sur ce point ANDERSON, Perry (1977), Sur le marxisme occidental, Maspero, Paris. 
Il s'agit d'un «État multinational ». On y parle de nombreuses langues : l'allemand, le hongrois et le tchèque, mais aussi le slovaque, le polonais, le russe, le roumain, l'italien, le yiddish, etc. On y pratique plusieurs religions : la catholique, la protestante, l'orthodoxe, la juive et la musulmane. Or la Double monarchie est menacée dans son existence même. Deux puissants facteurs menacent l'unité de l'empire, facteurs qui se renforcent mutuellement. Premier facteur dissolvant : «le principe de nationalité » que Baver résume ainsi : "Chaque nation doit former un État! Chaque État ne doit comprendre qu'une seule nation! $» .^{2}$ Le principe de nationalité cherche à faire coïncider État et Nation. La montée en puissance de ce principe au cours du 19ème siècle est un fait maintes fois constaté. Et il travaille en profondeur les nationalités de l'Empire. Plusieurs nations aspirent à devenir un État. Dans la perspective de ce principe, un État multinational apparaît comme " une survivance de temps révolus ${ }^{3}$ condamnée à court terme. Tout ceci n'est pas sans conséquence sur l'organisation du parti social-démocrate lui-même, parti qui, aux yeux de nombreux socialistes européens, passait pour être une "Petite Internationale » insérée dans la grande. Or le principe de nationalité divise la classe ouvrière de l'Empire, ce qui ne manque pas de profiter à la bourgeoisie. "Dans l'entreprise, dans le quartier, au parlement, tout semble opposer les deux communautés [allemandes et tchèques] pour lesquelles l'internationalisme prolétarien n'existe que sur le papier. Les sociaux-démocrates, bien qu'imbriqués dans ces batailles nationales ont conscience que les luttes nationales profitent aux bourgeoisies nationales et nuisent aux prolétariats $»{ }^{4}$ Fautil dès lors un seul et unique parti révolutionnaire pour toute l'Autriche-Hongrie ou fautil qu'à chaque nationalité corresponde un parti révolutionnaire distinct au risque de détruire I'unité de la classe ouvrière?

Deuxième facteur de décomposition : depuis la fin du 19ème siècle, de nombreux intellectuels, et parmi eux des marxistes, estiment que le capitalisme est entré dans une nouvelle phase de développement nommée « impérialisme ». La tendance d'un État à l'expansion n'est pas nouvelle. «Rome, fondée sur l'esclavage, faisait une politique coloniale et pratiquait l'impérialisme $»^{5}$ écrira Lénine quelques années plus tard dans sa brochure L'impéria-

2 BAUER, Otto (2017), La question des nationalités, Syllepse, Paris, p. 239.

3 Ibid, p. 240

4 PASTEUR, Paul, Pratiques politiques et militantes de la social-démocratie autrichienne, 1888 1934 (2003), Paris, Belin, p. 58.

5 LENINE, Oeuvres complètes (1960), Éditions sociales, Paris, Éditions du progrès, Moscou, $\mathrm{p}$. 280. 
lisme, stade suprême du capitalisme (1916). Néanmoins, il existerait un impérialisme nouveau, proprement capitaliste. Le petit capital, les entreprises familiales, en concurrence sur le marché, le marché lui-même, céderaient la place à de "grands monopoles» industriels et bancaires poussant les États à conquérir de nouveaux territoires pour exporter des capitaux et pour accaparer les matières premières nécessaires à la production à grande échelle. A ce moment, les nations occidentales se sont déjà partagées la quasi-totalité du globe. II n'y a plus guère de «terres vierges ». Dès lors, chaque conquête par une puissance se fait nécessairement au détriment d'une autre puissance. Les rixes entre nations européennes se multiplient dans les périphéries mondiales, les relations se tendent. La Grande guerre se profile à l'horizon. Dans ce contexte de rivalité impérialiste, chaque puissance instrumentalise le principe de nationalité chez ses adversaires dans l'espoir de les affaiblir. Les Empires multinationaux sont particulièrement exposés à ces tentatives de démembrement. Les Allemands par exemple ont tout intérêt à soutenir les aspirations nationales dans l'empire russe, par exemple celles des Polonais; tout en refusant ces mêmes aspirations dans le Reich. Le Tsar n'est pas en reste ; il pratique la même politique à l'égard de l'Allemagne ; à l'égard aussi de l'Autriche-Hongrie en convoitant les régions russophones et en soutenant les revendications nationales des Slaves du sud. L'Italie, quant à elle, soutient les velléités séparatistes des Italiens d'Autriche. Eł ceci n'est qu'un aperçu de l'ensemble.

$C^{\prime}$ est dans ce contexte que Otto Baver écrit La question des nationalités. II $s^{\prime}$ agit de résoudre l'équation suivante : satisfaire au principe de nationalité en préservant l'État multinational. II s'agit pour Baver d'adapter l'État aux nouvelles aspirations en conférant à chaque nationalité ce qu'il appelle « une autonomie nationale culturelle ». Et pour y parvenir, il convient $d^{\prime}$ abord d'examiner le concept de nation, sa genèse et son évolution, afin $d^{\prime}$ établir sa compatibilité ou son incompatibilité avec une structure politique multinationale.

Les perspectives politiques proposées par Baver ne parviendront pas à endiguer ce qui, rétrospectivement, peut apparaître comme la disparition nécessaire et inéluctable de l'Autriche-Hongrie. Mais l'investigation théorique sur la nation a peut-être gardé quelque intérêt pour nous aujourd'hui. Dans la préface à la seconde édition de l'ouvrage, datant de 1924, Otto Baver écrit : "L'histoire a laissé loin derrière elle le programme politique que je défendais en 1907 pour la solution du problème des nationalités en Autriche-Hongrie ». Mais il ajoute : "ce qui constitue le noyau même de l'ouvrage, c'est ma tentative d'appréhender les nations modernes avec les moyens de la conception marxiste de l'histoire, comme des communautés de caractères issues de communautés de destin. Et cette partie de mon 
livre ne me semble aujourd'hui aucunement vieillie $"{ }^{6} C^{\prime}$ 'est à expliciter ce «noyau » de l'ouvrage que ces pages vont s'employer, en laissant de côté, donc, les développements qu'Otto Bauer consacre à «l'autonomie nationale culturelle».

\section{Le CARACTÈre NATIONAL}

«La nation est l'ensemble des hommes liés par la communauté de destin en une communauté de caractère $" .^{7}$ La définition peut surprendre au premier abord. La nation est définie sans mentionner l'État. Et il n'est pas explicitement fait référence à un territoire. La nation est une population, un ensemble d'hommes, mais pas la population d'un territoire économique. Baver déplore des confusions terminologiques. Ainsi l'expression "protéger le travail allemand » est inadéquate; quand on l'utilise, " on ne pense pas au travail allemand en Autriche ou aux États-Unis, mais à celui qui est fourni dans la région économique allemande $» .^{8}$ Or pour Baver, dans une région économique peuvent coexister de nombreuses nationalités. Ces usages linguistiques sont compréhensibles seulement à partir du moment où le principe de nationalité - "Chaque nation doit former un État! Chaque État ne doit comprendre qu'une seule nation! »-s'est imposé au point de devenir un présupposé de la discussion théorique.

Pour Baver, ce qui caractérise les hommes liés en une nation, ce n'est ni le territoire, ni la subordination à l'État, mais la communauté de caractère. L'existence de nationalités se manifesterait d'abord dans la diversité des caractère nationaux. "On ne peut aborder la question de la nation, écritil, qu'à partir du concept de caractère national ». ${ }^{9}$ De quoi s'agit-il ? Le caractère national désigne "l'ensemble des caractéristiques physiques et morales qui distinguent une nation $d^{\prime}$ une autre $» .^{10}$ La nation commencerait donc à se donner d'abord dans une expérience.

Le caractère nationale est une donnée empirique, ${ }^{11}$ mais il est paradoxalement difficile à apercevoir. II ne se donne que dans certaines situations. La rareté relative de ces dernières explique la difficulté à concevoir le carac-

6 BAUER, Question, p. 45.

7 BAUER, Question, p. 199.

8 BAUER, Question, p. 240.

9 BAUER, Question, p. 66.

10 BAUER, Question, p. 66.

11 Une question laissée en suspend. Peut-on à partir de l'intuition de tel ou tel caractère national parvenir à une définition? A moins qu'on ne puisse dépasser le stade de l'intuition. 
tère national. Autrement dit, l'existence du caractère nationale n'est pas nécessairement accompagnée de la conscience de son existence. "Tant qu'un homme ne connaît que ses co-nationaux, il est conscient des divergences et non de son accord avec eux. Si je ne fréquente que des Allemands, si je n'entends parler que d'Allemands, je n'ai aucune occasion de me rendre compte que les hommes que je connais me ressemblent sur un point : par leur germanité ; je ne vois au contraire que les différences: il est Souabe, je suis Bavarois ; c'est un bourgeois, je suis un ouvrier ; il est blond, je suis brun ; il est grincheux, je suis enjoué. C'est seulement en faisant la connaissance de peuples étrangers que je prends conscience du fait qu'ils me sont étrangers, alors que je suis réunis à tous ceux que j'avais fréquenté jusqu'alors, et à des millions d'autres, par le lien de l'appartenance à la même nation. La connaissance de ce qui est étranger est le présupposé de toute conscience nationale ». ${ }^{12}$ Bauer ajoute un peu plus loin : "L'Allemand qui ne connaît que des Allemands et n'entend parler que d'Allemands ne peut pas prendre conscience de sa différence par rapport à d'autres nations, et pas davantage de son accord avec ses co-nationaux, de son appartenance à sa nation, il $n^{\prime}$ a pas de conscience nationale ${ }^{13}{ }^{13}$ Le caractère national n'apparaît donc pas immédiatement à la conscience des individus. II n'apparaît que par contraste, comme la couleur qui ne se révèle que sur un fond de couleur différente. "Transportons le premier Allemand venu en pays étranger, disons chez les Anglais; il se rendra tout de suite compte que ce sont d'autres êtres humains qui pensent, qui sentent autrement, qui, à un même stimulus extérieur, réagissent autrement que son entourage allemand habituel $»{ }^{14}$ La conscience du caractère nationale ne peut naître que dans un pays étranger ou dans la fréquentation régulière $d^{\prime}$ une autre nationalités dans son propre pays. Ce qui semblait naturel, ce qui semblait appartenir à tout homme, est alors relativisé.

\section{Caractère national et conscience nationale}

Cette distinction entre caractère nationale et conscience nationale est fondamentale pour Baver. Elle permet de faire la critique de « la théorie psychologique de la nation ». Pour celle-ci, la nation reposerait sur la conscience nationale, ainsi que sur la volonté d'appartenir à une même nation. C'est ainsi qu'on lit souvent -et à tort- la thèse défendue par Renan dans Qu'est-ce qu'une nation? : "L'existence d'une nation est (pardonnez-moi cette mé-

12 BAUER, Question, p. 203.

13 BAUER, Question, p. 205.

14 BAUER, Question, p. 66. 
taphore) un plébiscite de tous les jours " ${ }^{15} \|$ ne s'agit pas pour Baver de nier l'importance, par exemple politique, de la conscience et de la volonté $d^{\prime}$ appartenir. ${ }^{16}$ II veut seulement souligner le caractère dérivé et contingent de cette dimension psychologique. Le caractère national est le présupposé de la conscience nationale. Les compatriotes ne sont pas unis, en premier lieu, par la pensée un destin commun. Ils sont des co-nationaux quand bien même ils n'auraient ni la conscience ni la volonté d'appartenir. La nationalité ne suppose pas l'assentiment ou le consentement de l'individu, elle n'est pas un acte mais un fait.

\section{COSMOPOLITISME ET INTERNATIONALISME}

A la difficulté de percevoir le caractère national, il faut ajouter qu'il peut faire l'objet d'une dénégation théorique. On refuse de voir la diversité des caractères nationaux et la seule diversité nationale qu'on veuille bien reconnaître alors est celle des langues. Otto Baver écrit : "La diversité des caractères nationaux est un fait empirique qui n'est nié que par le doctrinarisme qui ne voit que ce qu'il veut bien voir, et ne voit donc pas ce que tout le monde voit ». La théorie, loin d'éclairer le monde, vient sélectionner dans la réalité seulement ce qui la conforte en se détournant de tout ce qui la remet en question. "Nous retrouvons cette opinion chez beaucoup de théoriciens, poursuit Baver, qui s'appuient sur la doctrine catholique. Elle a été reprise par l'humanisme de la philosophie bourgeoise des Lumières. Elle fut l'héritage de bon nombre de socialistes qui voulaient s'en servir pour consolider le cosmopolitisme prolétarien $»{ }^{17}$ Bauer critique à de nombreuses reprises ce cosmopolitisme «naif » qui nie la diversité des nationalités. Le cosmopolitisme fut une première réaction de la classe ouvrière à la domination de classe dans les pays capitalistes développés. Là, la bourgeoisie

15 La thèse de Renan est plus complexe en réalité que ne le laisse croire l'expression « conception française de la nation » que sa conférence est censée définir. Le plébiscite ne concerne que ceux qui ont un passé commun. La nation n'est donc pas réductible au consentement à des valeurs communes.

16 L'idée de nation-plébiscite semble généreuse puisqu'elle n'est pas exclusive. Il suffit de consentir à des valeurs pour y être inclus. Mais la générosité peut se renverser très vite en instrument d'exclusion. Soit le slogan de l'extrême-droite texane : «America, love it or leave it ». Celui qui ne revendique pas les valeurs se voit exclu de la nationalité ; comme si la nationalité résidait dans la conscience. Pour Baver, il faut partir du caractère national et non de la conscience. Être américain n'implique aucunement d'aimer l'Amérique. Et l'on ne peut déchoir quelqu'un de sa nationalité au prétexte qu'il ne plébiscite pas la nation.

17 BAUER, Question, p. 191. 
s'est accaparée la question nationale. Ainsi, en Allemagne, « le pouvoir de la nation pouvait-il être autre chose, aux yeux des ouvriers allemands, qu'un prétexte mensonger destiné à fonder la domination de leurs ennemis de classes ? ». ${ }^{18} \mathrm{~A}$ ce cosmopolitisme spontané, la social-démocratie oppose "la politique internationale du prolétariat de toutes les nations », 19 autrement dit l'internationalisme. ${ }^{20}$ II ne s'agit pas de nier l'existence des nations, ni d'appeler à leur dépassement. L'internationalisme insiste seulement sur la convergence des intérêts des ouvriers de nationalités différentes face au capital. Si les ouvriers autochtones et les ouvriers immigrés, par exemple les Tchèques en territoire allemand, ne se réunissent pas dans une même organisation syndicale pour lutter de front, alors la bourgeoisie aura toute latitude pour les opposer et embaucher à moindre coût.

L'internationalisme n'implique donc pas la négation de la diversité des caractères nationaux. Solidarité des intérêts et similitude des caractères sont deux questions absolument distincte. La différence des caractères n'exclut pas les convergences d'intérêts. Otto Baver écrit dans une note : " La question de savoir si l'ouvrier allemand a davantage de signes distinctifs communs avec le bourgeois allemand ou l'ouvrier français $n^{\prime}$ a rien à voir avec cette autre question : I'ouvrier allemand doit-il engager une politique de classe ou une politique nationale, doit-il s'unir aux prolétaires de tous les pays contre le capital international, ou avec le bourgeois allemands contre les autres peuples $\gg .^{21}$

\section{Le CARActère nAtional AU-DelÀ deS MYthes}

Une fois admise l'existence d'un caractère propre à chaque nationalité, il reste à le penser convenablement. Beaucoup de résistances intellectuelles à l'idée de caractère national viennent de ce qu'il est trop souvent mal conçu. Première difficulté : il est très courant $d^{\prime}$ ' attribuer au caractère national la stabilité $»{ }^{22}$ Ceux qui refusent son existence n'ont alors qu'à s'employer à

18 BAUER, Question, p. 372.

19 BAUER, Question, p. 374.

20 Marcel Mauss propose une distinction similaire dans "La nation ef l'internationalisme » (1920) : «L'Internationalisme digne de ce nom est le contraire du cosmopolitisme. II ne nie pas la nation. II la situe. Inter-nation, c'est le contraire d'a-nation. C'est aussi, par conséquent le contraire du nationalisme, qui isole la nation ». Disponible à cette adresse : http://classiques. uqac.ca/classiques/mauss_marcel/oeuvres_3/oeuvres_3_15/nation_et_inter.html

21 BAUER, Question, p. 67.

22 BAUER, Question, p. 69 
montrer qu'une telle stabilité n'est pas observable. Parler de caractère national, ce serait tomber dans l' « essentialisme », le «fixisme», nier l'histoire et ses transformations incessantes. La position de Baver est intéressante justement parce qu'elle disjoint identité et stabilité. "On a surtout eu tort d'attribuer au caractère national la stabilité : I'histoire démontre le contraire. [...] Aucune personne instruite ne niera que l'Allemand d'aujourd'hui a plus en commun avec les autres nations civilisées contemporaines qu'avec les Germains de Tacite. [...] La communauté de caractère unit les membres d'une nation au cours d'une période donnée, mais elle n'unit en aucune manière la nation contemporaine à ses ancêtres $d^{\prime} i l y$ a deux ou trois millénaires. Quand nous parlons du caractère national allemand, nous entendons par là les caractéristiques qui sont communes à tous les Allemands d'un siècle ou d'une décennie bien précise $»{ }^{23}$ Plus loin, il ajoute : les co-nationaux « ne sont pas liés par une conformité caractérielle de génération en génération $»{ }^{24}$ Le caractère national $n^{\prime}$ est donc pas une substance soustraite à I'histoire, il varie. II n'est pas un être persistant, un élément durable. Plus précisément, il faut lui accorder une stabilité mais toute relative, à l'échelle de la décennie ou du siècle.

Deuxième difficulté : la tendance au « fétichisme du caractère national $» .^{25}$ Le caractère national n'est pas « l'esprit » ou « l'âme » du peuple», ou encore « le génie du peuple ». Ces expressions définissent ce que Baver nomme «le spiritualisme national ». ${ }^{26}$ Pour ce dernier, le caractère national désigne " une force créatrice qui détermine l'histoire $~^{27}$ et qui expliquerait en dernier ressort le devenir d'une nation. II s'agit d'une conception métaphysique de la nation; métaphysique puisqu'elle nie finalement l'histoire elle-même, celle-ci n'étant que l'exécution d'un principe spirituel. Pour Baver, le caractère national n'a aucun pouvoir causale. Il est un effet que l'on se doit de décrire si l'on veut commencer à comprendre ce que c'est qu'une nation. "Le caractère national ne constitue pas une explication, il reste lui même à expliquer $»{ }^{28} \|$ est le produit d'une histoire, non son principe explicatif.

23 BAUER, Question, p. 67.

24 BAUER, Question, p. 188.

25 BAUER, Question, p. 193.

26 BAUER, Question, p. 71.

27 BAUER, Question, p. 193.

28 BAUER, Question, p. 69. 


\section{Communauté national de destin et identité de classe}

Le caractère national est donc à expliquer. Et cette explication réside dans la définition de la nation proposée ci-dessus : " La nation est l'ensemble des hommes liés par la communauté de destin en une communauté de caractère ${ }^{29}$ La cause de la communauté de caractère national est " la communauté de destin ». Une précision terminologique : le destin n'est pas la destinée au sens téléologique. Le destin est le cours historique, les aléas auxquels vont être soumis un groupe d'hommes. Si les compatriotes ont une communauté de caractère, c'est parce qu'ils ont une histoire partagée. Mais attention, cette histoire ne désigne pas l'ensemble du passé derrière eux. Il s'agit de l'histoire d'une période historique relativement courte (le siècle ou la décennie pour reprendre les exemples de Baver). Ce qui explique les variations du caractère national.

Pour préciser, Baver propose de distinguer «communauté de destin » et « identité de destin » (qu'il nomme aussi« similitude du sort »). ${ }^{30}$ L'identité de destin est susceptible, comme la communauté de destin, de produire des caractères communs. Otto Baver prend l'exemple de la classe. La classe est une communauté de caractère regroupant des individus de nationalités différentes $;{ }^{31}$ ces individus ont un air de famille, des comportements, des consommations, des manières de juger, etc. qui ont quelques similitudes. "L'ouvrier allemand a aussi des points communs avec ses camarades de classe de toutes les autres nations : c'est ce qui le rattache à la communauté internationale de caractère de sa classe. Le typographe allemand a indubitablement certains traits communs avec les typographes de tous les autres peuples : il fait partie d'une communauté professionnelle internationale $»{ }^{32}$ Affirmer cela, ce n'est pas prendre ses distances avec la définition de la classe proposée par Marx, c'est la compléter. La classe, pour Marx, est d'abord une réalité en soi, une position objective dans les rapports de production. C'est éventuellement aussi, à certaines conditions, une réalité pour soi, une appartenance reconnue par la conscience. Bauer intercale entre les deux, la classe comme communauté de caractère. "Les prolétai-

29 BAUER, Question, p. 199.

30 BAUER, Question, p. 177.

31 Outre la nation et la classe, "tous les peuples ont en commun des caractéristiques qui permettent de nous reconnaître tous en tant qu'êtres humains ».

32 BAUER, Question, p. 67. 
res de tous les pays possèdent des traits de caractères semblables. [...] La situation de classe identique a gravé dans le caractère de l'ouvrier allemand, anglais, français, russe, américain ou australien les mêmes traits : même combativité, même esprit révolutionnaire, même morale de classe, même volonté politique ${ }^{3} \cdot{ }^{33}$ Cette communauté de caractère procède de la position dans les rapports de production, elle est par exemple l'effet de la condition de salarié dans la grande industrie. C'est parce qu'ils sont soumis à un même sort qu'ils se ressemblent. Eł ce sort commun, c'est le développement capitaliste. Mais ces traits partagés, ces similitudes, n'impliquent absolument pas la conscience de classe, c'est-à-dire la conscience, non de traits, mais d'intérêts partagés.

On voit toute la distance avec la communauté de caractère national. Tous les co-nationaux ne sont pas soumis au même sort. Le développement du capitalisme n'affecte pas de la même façon l'ouvrier allemand et le bourgeois allemand. Ils n'ont pas le même destin, en quoi ils sont fort différents l'un de l'autre. Et pourtant, ils ont des traits communs en tant que compatriotes. ${ }^{34}$ "L'Allemand moyen se distingue de l'Anglais moyen, eussent-ils par ailleurs, en tant qu'hommes appartenant à la même classe ou à la même profession, beaucoup de points communs, et [...] deux Anglais se rejoignent par toute une série de caractéristiques, fussent-ils par ailleurs séparés par des différences individuelles ou sociales $»{ }^{35}$ Bref, le caractère de classe est

33 BAUER, Question, p. 177-178.

34 La distinction entre communauté de destin et identité de destin permet d'approfondir la compréhension de ce qu'est le caractère national. La classe est une communauté de caractère. Les ouvriers de nationalités différentes ont des traits relativement similaires bien qu'ils ne soient pas continuellement en relations, bien qu'ils ne parlent pas la même langue. De leur côté, les co-nationaux, qui interagissent les uns avec les autres en permanence, ne sont pas nécessairement soumis au même sort -bourgeois, paysans et ouvriers ont des vies forts différentes- du moins, leurs sorts respectifs ne se recouvrent pas totalement (bourgeois, paysans et ouvriers d'une même nation ont des vies différentes). Du fait d'un sort partiellement partagé (ils vivent les mêmes événements politiques ou sportifs, etc.) et de l'interaction constante, "I'Allemand moyen diffère de l'Anglais moyen, mais ressemble à tout autre Allemand moyen ». Mais Bauer ajoute : "Cette assertion n'a qu'une valeur générale relative. Ne connaissons nous pas tous des Allemands qui ne possèdent rien de ce qui est généralement considéré comme le caractère national allemand ? » (p. 188). Le caractère des individus étant déterminé par de multiples causes, il n'est pas étonnant que la multitude des combinaisons possibles de causes produisent des caractères différents. Beaucoup de co-nationaux se ressemblent, mais pas tous. Ce qui réunit l'ensemble, c'est « qu'une même force a exercé son influence sur le caractère de chaque individu ».

35 BAUER, Question, p. 66. 
fondé sur la soumission au même sort. Tout autre est la logique présidant à la production du caractère national. II se constitue dans la communauté de destin, non dans l'identité de destin.

\section{« L'INTERACTION MUTUELLE DES COMPATRIOTES "}

Qu'est-ce que Baver entend ici par « communauté »? "Communauté signifie, selon Kant, "interaction réciproque profonde » (Troisième analogie de l'expérience : Fondement de la communauté). Seul le destin vécu dans une interaction réciproque profonde et dans un rapport mutuel constant, forge la nation $» .^{36} L^{\prime}$ interaction est le concept central permettant de penser la communauté de destin. "II n'y a pas de nation sans l'interaction mutuelle des compatriotes ${ }^{37}$ sans " une communauté mutuelle de relations ${ }^{38}$ Dans une note consacrée à la distinction entre communauté et société, Baver écrit : je vois « le fondement de la communauté, dans ce que l'individu est, $d^{\prime}$ 'après son être physique et mental, le produit $d^{\prime}$ innombrables interactions entre lui-même et les autres individus unis en une communauté $»{ }^{39}$

Ainsi, $I^{\prime}$ « interaction constante dans la communication directe et indirecte ${ }^{4}{ }^{40}$ " la communauté continuelle de relations ${ }^{41}$ produit et conserve le caractère national. Les ouvriers de nations différentes, même s'ils ont des traits communs, n'engagent pas de telles relations. Quelle forme peut prendre cette interaction constante? Baver donne des exemples de relations étroites entre compatriotes appartenant pourtant à des classes différentes: " ils vivent dans la même ville, lisent aux murs les mêmes affiches, les mêmes journaux, participent aux mêmes événements politiques et sportifs, [...] il leur arrive aussi de se parler, soit directement, soit par l'entremise des différents intermédiaires $\gg .{ }^{42}$ Un autre exemple, qui relativise au passage l'importance du territoire pour la définition de la nation : " La séparation géographique [...] ne constitue pas un obstacle à la communauté nationale de caractère. L'Allemand d'Amérique qui reste influencé par la culture allemande -même si ce n'est plus que par voie du livre et du journal alle-

36 BAUER, Question, p. 177.

37 BAUER, Question, p. 184.

38 BAUER, Question, p. 179.

39 BAUER, Question, p. 191.

40 BAUER, Question, p. 178.

41 BAUER, Question, p. 179.

42 BAUER, Question, p. 178. 
mands- qui donne à ses enfants une éducation allemande, reste, malgré toutes les séparations géographiques, un Allemand $»{ }^{43}$ Les co-nationaux n'ont donc pas nécessairement une expérience identique, mais ils ont une expérience partagée : ils partagent des espaces communs (par exemple, les villes), des temps communs (tel ou tel événement), des lectures communes (les journaux), etc.

Sur ce point, les analyses de Baver anticipent celles de Benedict Anderson dans L'imaginaire national. L'ouvrage de Baver figure d'ailleurs dans la bibliographie de ce dernier livre. Anderson souligne, lui aussi, l'importance décisive des livres et des journaux dans la genèse de la nation moderne et de la conscience nationale. Pour Anderson, la nation est une communauté imaginaire " parce que même les membres de la plus petite des nations ne connaîtront jamais la plupart de leurs concitoyens : jamais ils ne les croiseront ni n'entendront parler d'eux, bien que dans l'esprit de chacun vive l'image de leur communion. [...] En réalité, au-delà des villages primordiaux où le face-à-face est de règle (et encore...), il n'est de communauté qu'imaginée ». ${ }^{44}$ La communauté nationale ne peut naître de la relation en face-à-face. Elle l'excède évidemment. Elle naît de ce que chaque compatriote possède une « image » de sa communion avec tous les autres. Ce qui va rendre cette image possible, c'est la diffusion des livres et des journaux, en d'autres termes, c'est l'essor du " capitalisme de l'imprimé ». ${ }^{45}$ "Ce qui, de manière positive a rendu les nouvelles communautés imaginables, c'est I'interaction à demi fortuite, mais explosive, entre un système de production et de rapport de production (le capitalisme), une technologie de communication (l'imprimé) et la fatalité de la diversité linguistique ». ${ }^{46}$

\section{Nation et langue}

Les exemples de Baver montrent l'importance de la langue pour la nation, importance qu'il ne faut cependant pas mal comprendre. Certes, "la nation est obligatoirement une communauté de langue $" .{ }^{47}$ Car sans langue commune, l'interaction ne saurait être constante. ${ }^{48}$ Mais la communauté de

43 BAUER, Question, p. 197.

44 ANDERSON, Benedict (2002), L'imaginaire national. Réflexions sur l'origine et l'essor du nationalisme, La découverte, Paris, p. 19.

45 ANDERSON, Imaginaire, p. 51.

46 ANDERSON, Imaginaire, p. 54.

47 BAUER, Question, p. 664. 
langue ne fait pas nécessairement une communauté de destin. Condition nécessaire donc mais pas suffisante : "la communauté de langue $n^{\prime}$ est pas en elle-même une garantie d'unité nationale ». ${ }^{49}$ Ainsi Espagnols et Sud-américains hispanophones constituent plusieurs nations distinctes. La langue fait partie de ce que Baver nomme des éléments de « deuxième ordre » dans la définition de la nation. "Je ne nie donc pas que la nation est communauté linguistique, mais je recherche derrière la langue, ce qui la produit, suscite ses mutations, déterminent les bornes de sa validité $» .{ }^{50}$ Ainsi, finalement, même si « le langage est la condition de toute relation étroite » (180), c'est I'histoire partagée, non la langue, qui définit la communauté de destin.

\section{Communauté de destin et matérialisme historiQue}

Cette histoire est définie dans les termes mêmes de Marx. Baver écrit : « Les conditions dans lesquelles les hommes produisent leur subsistance et se répartissent le fruit de leur travail détermine le destin de chaque peuple; c'est sur la base d'un certain mode de production et de distribution des moyens de subsistance qu'apparaît une certaine culture intellectuelle $»{ }^{51}$ L'interaction profonde des compatriotes se déploie donc dans le cadre d'un mode de production qui lui donne ses traits les plus généraux.

Cette thèse -la base économique détermine en dernière instance la communauté de destin et donc le caractère national- permet de formuler une hypothèse relative à la variabilité du caractère national. Baver écrit : "Aucune personne instruite ne niera que l'Allemand d'aujourd'hui a plus en commun avec les autres nations civilisées contemporaines qu'avec les Germains de Tacite $»{ }^{52}$ Nous avons plus en commun avec nos contemporains d'autres nationalités qu'avec nos propres ancêtres. A condition, bien entendu, qu'on se donne une échelle de temps assez longue. ${ }^{53}$ Comment expliquer

48 A ce titre, si l'on ne confond pas État et nation, la Suisse n'est pas une nation, mais un État multinational.

49 BAUER, Question, p. 180.

50 BAUER, Question, p. 665.

51 BAUER, Question, p. 89.

52 BAUER, Question, p. 67.

53 Georges Orwell écrit dans Le lion et la licorne : «Qu'est-ce que l'Angleterre de 1940 peut bien avoir en commun avec celle de 1840 ? Mais aussi, qu'avez-vous de commun avec l'enfant de cinq ans dont votre mère garde précieusement une photographie? Rien, si ce n'est que vous êtes la même personne ». Analogie entre peuple et individu mise de côté, on peut noter que l'échelle de temps envisagée est relativement courte. Elle correspond à la période de stabilité 
ce paradoxe? Les différentes communautés de destin contemporaines sont toutes déterminées en dernière instance (ce qui ne signifie pas « déterminées exclusivement ») par un même mode de production : le capitalisme. L'existence des Allemands d'hier et d'aujourd'hui se déploie en revanche sur des bases économiques différentes. L'histoire est donc discontinue. Cette discontinuité, qui est celle des modes de production, explique à la fois la relative stabilité du caractère national sur une petite échelle de temps et sa variation à long terme.

Voici ce que Bauer écrit du passage de l'agriculture traditionnelle à l'agriculture contemporaine en Allemagne. ${ }^{54}$ "Toutes ces immenses transformations provoquent $d$ 'une part un bouleversement complet de la population, à la fois géographique et professionnel, et d'autre part un changement radical de la position économique et donc de la psychologie du paysan ».55 Intuition pénétrante d'une réalité souvent absente de la pensée critique : les effets psychologiques de la modernisation capitaliste. Baver ajoute : « La population rurale a été déracinée par le capitalisme, arrachée du sol auquel elle était fixé depuis la sédentarisation du peuple, chassée des frontières étroites du territoire du village ${ }^{56}$ Et de conclure : «Ne sommes-nous pas devenus $d^{\prime}$ autres hommes parce que nous avons été déracinés du sol que nous cultivions, des forêts et des champs que nous sillonnions, et déportés dans les grandes villes avec leurs casernes de rapport, dans les zones industrielles avec leur suie et leur fumée, où une dernière fleur et un dernier arbre étouffent dans l'air imprégnée de charbon! $» .{ }^{57}$

Ceux qui définissent le caractère national par sa permanence, son identité dans le temps, oublient les ruptures historiques que constitue le passage d'un mode de production à un autre, ou plutôt, le passage dans une structure sociale donnée de l'hégémonie d'un mode de production à l'hégémonie $d^{\prime}$ un autre. Le changement économique est radical : il bouleverse la population jusque dans ses structures psychiques les plus profondes. Bien sûr, toute l'historicité ne se ramène pas au mode de production. II n'en reste pas moins que ses développements sont structurants en dernier instance jusqu'au niveau psychologique. ${ }^{58}$

relative d'un caractère national pour Baver (quelques décennies à un siècle). Pourrait-on répondre de la même manière à la question : "Qu'est-ce que l'Angleterre de 1940 peut bien avoir en commun avec celle de 1440 ? ».

54 WEBER, Eugen (2011), La fin des terroirs (1870-1914), Pluriel, Paris. Le livre d'Eugen Weber décrit le même processus pour la France.

55 BAUER, Question, p. 153-154

56 BAUER, Question, p. 154 


\section{Nation, nature et CUlture}

La nation est donc de part en part histoire. Néanmoins, elle ne semble pas dénuée d'une certaine forme de naturalité dans l'ouvrage de Baver. Le second chapitre de l'ouvrage s'intitule ainsi : "La nation comme communauté de nature ». L'idée d'une naturalisation de la nation peut à bon droit inquiéter. Dans la préface à la première édition française du livre, Claudie Weill souligne "l'ambiguitié ${ }^{59}$ de ce thème pouvant susciter de graves contresens. La perspective de Baver n'est pourtant pas dépourvue d'intérêt.

Sans exclure la nature de sa réflexion, il entreprend la critique du « matérialisme national » qui fait de la nation une substance matérielle ancestrale se transmettant de génération en génération. Pour le matérialisme national, «c'est la capacité d'enfanter et d'engendrer de la race qui décide de l'histoire du peuple ; le maintien de la pureté du sang, le mélange des germes de communautés d'origine différente, voilà les événements vraiment marquants de l'histoire universelle $» .{ }^{60}$

L'originalité de Baver vient de ce qu'il décide de jouer Darwin et Marx contre le matérialisme national. Ce dernier se revendique d'une vision mythique de la nature, d'une biologie périmée par la théorie de l'évolution. Des traits héréditaires se transmettent dans l'histoire; c'est un fait. Mais avec la théorie de l'évolution, la question devient : pourquoi ces traits-là, et non pas d'autres? Et c'est alors l'histoire qu'il faut interroger et non plus la physiologie. Baver écrit : "Les traits hérités d'une nation ne sont que la sédimentation de leur passé, pour ainsi dire, leur histoire figée ». "Q1 "Qu'un peuple ou un autre soit particulièrement combatif peut effectivement provenir d'une hérédité conditionnée par la physiologie. Mais pourquoi la combativité se transmet-elle ici ? Peut-être parce qu'il y a des siècles, les ancêtres de ces peuples menaient une vie nomade et guerrière, que les moins combatifs

57 BAUER, Question, p. 169

58 Les formules de Baver peuvent être rapprochées des travaux de Lucien Sève sur les formes historiques d'individualité : "Sont dites formes historiques d'individualité (en abrégé $\mathrm{FHI}$ ) toutes les façons d'être induites chez les individus par une formation sociale donnée ». "Bien entendu, le fait naturel de l'individualité est autonome, avec ses contraintes biologiques comme d'être sexué ou de parcourir le cycle fini des âges de la vie, avec ses caractéristiques neuropsychiques communes à tous ou propres à chacun : I'individualité psychique est bien tout autre chose qu'une superstructure du tout social. Mais en même temps il n'est rien dans les individus, fût-ce le plus directement biologique, qui ne porte en son essence même les marques spécifiques de la formation sociale dont ils sont membres ». SEVE, Lucien (2015), Pour une science de la biographie, suivi de Formes historiques d'individualité, Éditions sociales, Paris, p. 74. 
ont été progressivement écartés du processus de reproduction, et que seuls les plus aptes au combat ont donc pu se reproduire. La combativité héritée par un peuple est donc la sédimentation de son histoire des siècles passés, la résultante des conditions dans lesquelles il a cherché sa subsistance ${ }^{62}$ Baver conclut : "Les conditions dans lesquelles un peuple produit ses moyens de subsistance règlent la sélection ». "Dans les traits de caractère dont héritent les générations suivantes se reflètent donc les conditions de productions des générations précédentes $"{ }^{63}$ Ainsi, même en admettant une naturalité au moins partielle de la nation, ce n'est pas l'hérédité qui constitue l'explication en dernier ressort mais le mode de production et le processus de sélection qui lui est associé. ${ }^{64}$

Otto Baver relativise d'une autre manière l'influence de l'hérédité biologique. II propose une expérience de pensée : «Supposons qu'une violente catastrophe fasse disparaître tous les Allemands, et que de tout le peuple allemand ne subsiste que quelques enfants en bas âge. Supposons qu'en même temps qu'eux, tous les trésors culturels allemands, ateliers, écoles, bibliothèques et musées soient anéantis. $D^{\prime}$ heureuses circonstances permettent à ce malheureux peuple de grandir et d'en fonder un nouveau. Ce peuple sera-t-il allemand? Bien sûr, les enfants sont pourvus de toutes les dispositions héréditaires du peuple allemand, qui ne sont pas perdues. Mais la langue qu'ils vont lentement inventer ne sera pas une langue allemande, les mœurs, le droit, la religion, la science, l'art, la poésie, tout cela, ce nouveau peuple va devoir se le recréer dans un long processus de développement, et ces hommes qui vivront dans des conditions complètement transformées auront de tout autres traits de caractère que les Allemands d'aujourd'hui ». ${ }^{65}$ La nation est bien transmission (pas plébiscite), mais transmission de ce que Baver nomme « biens culturels »: langue religion, science, art, etc. La nation est donc en premier lieu une communauté de culture. La catastrophe

59 BAUER, Question, Introduction de Claudie Weil, p. 28.

60 BAUER, Question, p. 77

61 BAUER, Question, p. 85

62 BAUER, Question, p. 83

63 BAUER, Question page 83

64 En ce début du 20ème siècle, les marxistes considèrent que les œuvres de Darwin et de Marx, loin de s'opposer, se complètent. Le darwinisme social leur apparaît pour ce qu'il est : une falsification des travaux de Darwin. Voir par exemple, PANNEKOEK, Anton (2012), Darwinisme et marxisme, Arkhê édition, Paris.

65 BAUER, Question, p. 87 
mentionnée détruit la nation alors même que la transmission biologique héréditaire, bien que menacée, continue.

Mais Bauer va plus loin. Le rapport entre nature et nation varie au cours de I'histoire. Thèse importante : plusieurs types de nationalité se succèdent. ${ }^{66}$ Historiquement, la nation fut d'abord liée à la communauté de nature. Ainsi écrit-il des Germains : "C C'est principalement l'origine commune qui fait des Germains de cette époque une nation $"{ }^{67}$ Mais l'origine commune, loin de définir la nation, ne caractérise que ce stade primordial. Ainsi, le développement historique "détruit l'ancienne communauté de nature sur laquelle reposait encore la communauté de culture germanique $»{ }^{68}$ Du fait de l'accroissement de la population, les tribus se dispersent. L'isolement territorial différencie de plus en plus des tribus relativement similaires à l'origine. Soumises à des conditions d'existence différentes, elles se mettent à produire différemment, les traits sélectionnés et transmis diffèrent eux aussi. Et à l'isolement s'ajoute "le mélange avec des peuples étrangers ", 69 ici avec les Celtes.

La « désintégration de la nation qui repose sur la communauté d'origine » va céder la place à d'autres formes nationales. La nation allemande qui naîtra ensuite, selon Bauer, ne devra rien à cette communauté d'origine des Germains. Ce qui fera la nation allemande, c'est « une toute nouvelle forme de culture commune $"{ }^{70}$ la culture de la classe dominante, la chevalerie, qui fera effort pour mettre un terme à la différenciation extrême qui succéda à la désintégration de la nation germaine.

II n'est pas exclu que pour certains peuples la communauté de nature et la communauté de culture continuent de coïncider. ${ }^{71}$ Mais ce n'est qu'un scénario possible. II existe "des nations sans communauté d'origine qui

66 Bauer s'intéresse aux Germains et aux Allemands. Peutêtre faut-il se garder de généraliser cette succession. Néanmoins, cette précaution n'enlève rien à l'idée d'une pluralité des types de nationalités. L'unité de ces différents types étant : la communauté de caractère fondée sur la communauté de destin.

67 BAUER, Question, p. 92

68 BAUER, Question, p. 102

69 BAUER, Question, p. 103

70 BAUER, Question, p. 114

71 Eric Hobsbawm écrit dans « Qu'est-ce qu'un conflit ethnique ? » : Notons que l'homogénéité ethnique est extrêmement rare parmi les entités politiques d'une dimension suffisante pour accéder au rang d'État, même de nos jours. Parmi les 180 États membres des Nations unis, il n'y en a guère plus d'une douzaine dont on pourrait dire qu'en gros ils correspondent à une langue ou à une race ou ethnie ». 
sont fondues en une unité profonde par la seule force de la communauté de culture $» .^{72}$ " La nation peut donc reposer sur la communauté d'origine, mais sans nécessité aucune, tandis que la simple communauté d'origine, mais sans nécessité aucune, tandis que la simple communauté $d^{\prime}$ 'origine ne forme rien qu'une race, jamais une nation $\gg .^{73}$

\section{L'AVENIR DES NATIONS}

A la différence de nombreux marxistes de son temps, Bauer ne se situe pas dans l'horizon d'une «fusion des nations ». Pour Kautsky, mais aussi Lénine ou encore Pannekoek, les nations sont des réalités transitoires vouées à disparaître à plus ou moins long terme. Kautsky écrit dans la revue Die Neue Zeit que le rapprochement des nations est produit « par la force omnipotente de l'avantage que cette association apporte à tous. De façon indolore, les nations se confondent les unes dans les autres, à peu près à la manière de la population rhéto-romane des Grisons, qui, imperceptiblement et sans rechigner, se germanise peu à peu parce qu'elle trouve plus avantageux de parler une langue que tout le monde comprend dans un vaste rayon qu'un langage qui n'est parlé que dans quelques vallées $» .^{74}$ Lénine, même s'il soutient les revendications nationales des nations opprimées, s'inscrit dans cette horizon. Le prolétariat « soutient tout ce qui aide à effacer les distinctions nationales et à faire tomber les barrières nationales, tout ce qui rend la liaison entre nationalités de plus en plus étroite, tout ce qui mène à la fusion des nations ».

Pour Baver, au contraire, l'avenir socialiste verra « la différenciation croissante des nations $»{ }^{75}$ Non pas leur multiplication, mais l'affirmation toujours plus nette de leur singularité. Bien entendu, les échanges internationaux produisent un certain nivellement : "Tout progrès technique est approprié en quelques années par le monde entier, toute modification du droit chez les peuples voisins est étudiée et imitée, tout courant scientifique ou artistique influence les peuples civilisés du monde entier ». ${ }^{76}$ Mais cela ne signifie pas que les nations deviennent identiques. Chaque nouveauté est incorporée à la communauté de destin ; pas d'enregistrement mécanique mais une

72 BAUER, Question, p. 184.

73 BAUER, Question, p. 195.

74 KAUTSKY, Karl (1887), « La nationalité moderne », in HAUPT, Georges; LÖWY, Michael et WEIL, Claudie (1974), Les marxistes et la question nationale, 1848-1914, Paris, Maspero.

75 BAUER, Question, p. 171.

76 BAUER, Question, p. 171. 
" digestion intellectuelle ». ${ }^{77} \mathrm{Ce}$ qui est reçu est adapté, accommodé, à l'être national, lequel persiste donc dans sa singularité.

Néanmoins, cette thèse $n^{\prime}$ exclut pas que certains groupes nationaux -tout ou partie- périclitent et finissent par être assimilés par d'autres nationalités. La question se pose lorsqu'une nationalité se trouve en minorité. Baver pense aux Tchèques dans les territoires allemands de l'Empire et aux Juifs. Placée dans ces conditions, une nationalité peut se maintenir mais pas nécessairement. Elle peut s'assimiler (le mot est de Baver) à la nation majoritaire, se fondre en elle : c'est le cas de la nation juive pour Bauer qui s'assimile à la nation allemande. Elle peut, à certaines conditions se maintenir dans sa singularité (c'est le cas des Tchèques pour Baver).

\section{Otto Bauer, multiculturaliste ?}

Baver a pu apparaître à certains égards comme un précurseur du multiculturalisme. Raison de le condamner pour les uns, raison de le lover, voire de le republier, pour les autres. C'est l'intention affichée par exemple dans l'avant-propos de la réédition chez Syllepse de La question des nationalités. Baver donnerait des outils pour penser le présent, en particulier son concept $d^{\prime}$ " autonomie nationale culturelle ». Reconnaître aux nationalités de l'Empire une autonomie culturelle, c'était leur confier le soin d'administrer leurs écoles, leurs musées et leurs administrations, leur donner la liberté d'organiser à leur guise la transmission de leurs biens culturels. Cette perspective politique -sur laquelle nous avons choisi de ne pas nous centrer-serait prometteuse dans les sociétés capitalistes les plus avancées. «La France urbaine, dit l'avant-propos, est à la fois «noire», " arabe » et «blanche» -et toujours genrée cela va de soi- et sa classe travailleuse a toujours été " plurinationale ». ${ }^{78}$ Laissant de côté la France qui n'est pas urbaine (où les problèmes se posent différemment que dans les métropoles), laissant de côté aussi les Français ou immigrés venus d'Asie (fautil dire « Jaunes »?), ils vont directement à la conclusion politique : "une république autogérée devrait sans aucun doute intégrer dans son édifice institutionnel les différentes chambres d'intérêts particuliers de manière notamment à ce que les « minorités » ne soient pas confinées en toutes choses à rester démocratiquement minoritaires $»{ }^{79}$

77 BAUER, Question, p. 173.

78 BAUER, Question, avant-propos de Didier Epsztajn, Patrick Le Tréhondat, Patrick Silberstein, p. 13.

79 BAUER, Question, avant-propos de Didier Epsztajn, Patrick Le Tréhondat, Patrick Silberstein, p. 18. 
II ne s'agit pas de porter un jugement sur le projet de république présenté ici. Il s'agit, plus modestement, d'en appeler à une lecture prudente du livre de 1907. Les perspectives mentionnées ci-dessus doivent beaucoup plus aux théories multiculturelles venues d'Amérique du nord à la toute fin du XX ème siècle qu'à l'ouvrage d'Otto Baver. Les différences sont gigantesques et les minimiser peut conduire à de sérieux contresens sur La question des nationalités.

Pour Otto Baver, toutes les groupes nationaux de l'Empire ne peuvent pas prétendre à l'autonomie nationale culturelle. II faut pour cela remplir plusieurs conditions qui renvoient toutes à la définition de la nation comme communauté de destin. Faut-il accorder aux Juifs par exemple, à l'instar des Tchèques, l'autonomie nationale culturelle? Pour Baver, les immigrés tchèques en territoire allemand ne peuvent former une communauté de destin avec les Allemands en particulier parce qu'ils ne parlent pas la même langue. En effet, il faut se rappeler que « la nation est obligatoirement une communauté de langue ${ }^{80}$ Sans langue partagée pas de nation. Des individus de nationalité tchèque peuvent apprendre l'allemand, ils peuvent s'assimiler alors à la nation allemande. Mais ils sont alors immédiatement remplacé par de nouveaux arrivants qui maintiennent la population tchèque dans son originalité. Les Juifs, pour Baver, ne sont pas comparables aux Tchèques. "La question de la langue n'existe pas pour les Juifs. Car vivant au milieu d'autres peuples et entrant en contact économique toujours plus étroit avec eux, il leur faut bon gré mal gré parler la langue de la majorité. Le Juif qui travaille dans un atelier avec des Polonais, achète ou vend à des Polonais, sera capable aussi de défendre ses droits en polonais devant les autorités ou les tribunaux $» .^{81}$ Une des conditions de la communauté de destin, la langue commune -ici le polonais-, est réunie dans le cas des Juifs. Et dans les territoire allemands de l'empire, les Juifs sont des Allemands de confession juive.

Or il y a fort à parier que la France «noire », " arabe » et « blanche », évoquée dans l'avant-propos, parle le français. A moins de construire des ensembles à la cohérence douteuse regroupant des populations arrivées hier et ne parlant pas la langue du pays et des gens installés en France depuis plusieurs générations déjà. Si l'on s'en tient à ceux qui parlent le français, il semble plus conforme au texte de Baver de dire qu'ils partagent une même "identité de destin », une communauté de caractère issue de l'expérience partagée par exemple d'un même type d'habitat, d'un même

80 BAUER, Question, p. 664

81 BAUER, Question, p. 451. 
rapport au travail, à la police, etc. Mais ces traits déterminés par la similitude du sort ne sont qu'une partie des traits individuels. Ils côtoient ceux engendrés par la communauté national de destin. Que les intéressés n'aient pas conscience de ces traits partagés avec les Français d'une autre couleur, est tout à fait possible. Néanmoins, la conscience nationale, pour Baver, ne fonde pas la nationalité.

Bref, les analogies entre les positions de Baver et les formes contemporaines de multiculturalisme doivent être maniées avec précaution.

\section{BibLIOGRAPHIE}

ANDERSON, Benedict (2002), L'imaginaire national. Réflexions sur l'origine et l'essor du nationalisme, La Découverte, Paris.

ANDERSON, Perry (1977), Sur le marxisme occidental, Maspero, Paris.

BAUER, Otto (2017), La question des nationalités, Éditions Syllepse, Paris.

HAUPT, Georges, LÖWY, Michael et WEIL, Claudie (1974), Les marxistes et la question nationale, 1848-1914, Maspero, Paris.

HOBSBAWM, Eric (1993), "Qu'est-ce qu'un conflit ethnique? », in Actes de la Recherche en Sciences Sociales, vol. 100, pp. 51-57.

ORWELL, Georges (1982), The Lion and the Unicorn: Socialism and the English Genius, Penguin Books, London.

MAUSS, Marcel (1920), "La nation et l'internationalisme », http://classiques.uqac.ca/classiques/mauss_marcel/oeuvres_3/oeuvres_3_15/nation_et_inter.html

LENINE, Oeuvres complètes (1960), Éditions Sociales / Éditions du progrès, Paris / Moscou.

PANNEKOEK, Anton (2012), Darwinisme et marxisme, Arkhê édition, Paris.

PASTEUR, Paul (2003), Pratiques politiques et militantes de la social-démocratie autrichienne, 1888-1934, Belin, Paris.

SEVE, Lucien (2015), Pour une science de la biographie, suivi de Formes historiques d'individualité, Éditions Sociales, Paris.

WEBER, Eugen (2011), La fin des terroirs (1870-1914), Pluriel, Paris. 